\title{
Mediennutzung in Zeiten von Corona
}

\author{
Die Geschichte von 2 Pandemien
}

Korrespondenzadresse

Prof. Dr. Dr. Manfred Spitzer

Universität Ulm

Abteilung für Psychiatrie

Leimgrubenweg 12-14

87054 Ulm

Deutschland
Bibliografie

DOI https://doi.org/10.1055/a-1193-8248

Nervenheilkunde 2020; 39: 698-703

(C) 2020. Thieme. All rights reserved.

Georg Thieme Verlag KG, Rüdigerstraße 14,

70469 Stuttgart, Germany

ISSN 0722-1541
Schon vor der Corona-Krise hatte die weltweite Nutzung digitaler Bildschirmmedien Ausmaße angenommen, die aufgrund der bekannten medizinisch-wissenschaftlichen Fachliteratur als besorgniserregend einzustufen war. Insbesondere für Kinder und Jugendliche, deren Gehirne besonders aufnahmefähig sind und zu deren Entwicklung adäquate Erfahrungen innerhalb ihrer gelebten Kultur erfolgen müssen - wie beim Spracherwerb, nur eben viel breiter - ist die übertriebene Nutzung digitaler Bildschirmmedien nachweislich schädlich: nicht nur für die Gesundheit, sondern auch für Bildung und soziale Entwicklung. Sogar die Säulen des gesellschaftlichen Zusammenhalts in unserer demokratischen Gesellschaft - Wahrheit, Vertrauen, Solidarität - sind durch die nachgewiesenen Auswirkungen digitaler Bildschirmmedien bei übertriebenem Gebrauch in Gefahr:

- Lügen werden in sozialen Online-Medien (z. B. Twitter) schneller, weiter und tiefer verbreitet als Wahrheit, wie eine im Fachblatt Science publizierte Studie zu 126000 Tweets, die 4,5 Millionen Mal verbreitet wurden, gezeigt hat [48].

- Weil wir anstatt nach dem Weg oder der Uhrzeit eine fremde Person zu fragen, nur noch auf unser Smartphone schauen, haben wir weniger glückende kleine Kontakte mit fremden Menschen. Diese sind jedoch der Nährboden unseres gegenseitigen Grundvertrauens, wie Soziologen nachweisen konnten [23].

- Je mehr Stunden täglich Jugendliche vor Bildschirmen verbringen und damit weniger reale Kontakte haben (nur durch diese kann man Empathie lernen, auch das wissen wir), desto weniger Mitgefühl haben diese Jugendlichen für ihre Eltern und Freunde, wie in den beiden weltweit größten Längsschnittstudien hierzu nachgewiesen wurde [32].

- Der gleiche Mechanismus - die Medialisierung unserer Welt - bewirkt in jedem Lebensalter mehr soziale Isolation und Einsamkeit - mit einer epidemiologisch an zunächst gut 300000 [16] und später noch einmal bei knapp 3,5 Millionen [17] Menschen nachgewiesenen deutlich gesteigerten Sterblichkeit.

- Um die Zuschauer am Bildschirm zu halten, zeigt YouTube, von dessen Computern 70-80\% der gesehenen Videos vorgeschlagen wird, zunehmend radikale Inhalte, egal, worum es geht. Daher ist die Radikalisierung der Bevölkerung Bestandteil des Geschäftsmodells von YouTube. Die Videos dieser Plattform wurden schon vor Corona von einem großen Teil der Menschheit zusammengenommen täglich für eine Milliarde Stunden angeschaut. Die politischen Folgen von 700 bis 800 Millionen Stunden Radikalisierung der Menschheit täglich hören und sehen wir täglich in den Nachrichten.

- Hinzu kommt: Mehr Hass-Sprache führt nachweislich zu mehr Hass-Gewalt [19, 26, 50]. Auch das zeigen die Nachrichten täglich.

- Die Anonymität im Internet bewirkt, dass sich viele Menschen dort enthemmter und aggressiver gegenüber Mitmenschen verhalten (Bullying, Mobbing) - mit psychischen [42] und gesundheitlichen Folgen [35] für die Betroffenen. Und das sind vor allem Kinder und Jugendliche, von denen $50 \%$ über ihr Smartphone gemobbt werden und nahezu alle im Alter von 13 Jahren bereits mindestens einmal sexuell belästigt wurden.

- Die Möglichkeit und Wirklichkeit von Manipulation großer Menschenmassen wurde experimentell im Hinblick auf Emotionen [22] ( $\mathrm{n}=700000)$, Kaufentscheidungen [25] $(n=3500000)$ und Wahlentscheidungen [2] $(n=61000000)$ nachgewiesen.

- Die Gefährdung unserer Privatsphäre ist zwar bekannt, ist aber den meisten Menschen nachweislich egal [3, 31], mit Ausnahme der Deutschen [51]. Die unabhängigen Bewertungs- und Entscheidungsfindungsprozesse, welche eine Grundlage unserer Demokratie sind, können aber nur funktionieren, wenn es Privatheit gibt - auch dies wurde empirisch nachgewiesen [36].

Vor diesem Hintergrund ist die Zunahme der Nutzung digitaler Bildschirmmedien seit dem Beginn der Corona-Pandemie und vor allem seit dem Lockdown (Kontaktbeschränkungen, Abstandsregeln und die Schließung von Kitas und Schulen, Spiel- und Sportplätzen, vieler Geschäfte sowie privater und öffentlicher Institutionen) von besonderem wissenschaftlichen, aber vor allem auch von ganz allgemeinem gesellschaftlichen Interesse - weltweit. Denn die globale Corona-Pandemie trifft eben auf die weltweite Verbreitung und Nutzung digitaler Bildschirmmedien und auf deren tägli- 
che Nutzung für durchschnittlich mehrere Stunden, was zur Folge hatte, dass am 20. März 2020 nach entsprechender Aufforderung der EU-Kommission die Streaming-Dienste Netflix und YouTube ihre Bildqualität senken mussten, um einen Zusammenbruch der Datennetze zu verhindern [5]. ${ }^{1}$

Die zur Infektionsvermeidung während des Lockdowns geforderte körperliche Distanzierung wurde zunächst leider als „soziale Isolation“ („social distancing“) bezeichnet und auch so umgesetzt [41]. Diese führt in vielen Fällen zu mehr Einsamkeit [37], und die wiederum wirkt sich negativ auf die Funktion des Immunsystems aus [37, 40]. Genau dies ist jedoch unser wichtigster Schutzfaktor gegenüber der Krankheit. Müssen wir also nicht sogar Einsamkeit bekämpfen, um uns und unsere Kinder vor Corona zu schützen? Und folgt daraus „Netflix kostenlos für alle?“, was in Deutschland seit dem 1. September 2020 tatsächlich (bei im Vergleich zu zahlenden Nutzern eingeschränktem Angebot) gilt. ${ }^{2}$

Halten wir fest: Die Corona-Krise hat zu einer deutlich höheren Nutzung digitaler Medien geführt. So negativ die weltweiten wirtschaftlichen Folgen der Krise auch waren, die reichsten Firmen der Welt - Amazon, Apple, Facebook, Google und Microsoft - haben enorm profitiert. Was bedeutet dies für die Kinder und Jugendlichen in Deutschland?

Die Deutsche Angestelltenkrankenkasse (DAK) gib schon seit längerer Zeit beim forsa Institut für Politik- und Sozialforschung Befragungen zur Mediennutzung von Kindern und Jugendlichen in Auftrag [7-10]. Dadurch wurde es möglich, durch Vergleich im Längsschnitt die Folgen der Corona-Krise für die Mediennutzung zu untersuchen. Hierzu wurde eine vom 13. bis 17. September 2019 stattgehabte Befragung von 1221 Kindern und Jugendlichen (Alter: 10 bis 17 Jahre; Mittelwert: 13,0 Jahre) sowie je eines Elternteils (Alter: 28-75 Jahre; Mittelwert: 46,2 Jahre) zur Nutzung von digitalen Spielen und sozialen Online-Medien nochmals im Zeitraum vom 20. bis 30. April 2020 durchgeführt. Die zweite Befragung fand also einen Monat nach dem deutschen Lockdown bzw. etwa 2 Wochen nach dem Scheitelpunkt der Pandemie in Deutschland statt. An dieser Wiederholung beteiligten sich 824 (67,5\%) der im Herbst zuvor befragten Familien, sodass ein Vergleich der Daten im Längsschnitt möglich war. Zwar gibt es mittlerweile bereits einige Beobachtungen zur gestiegenen Mediennutzung aufgrund der Corona-Pandemie, weil die Schule ausfiel und auch sonst nicht viel los war bzw. sein durfte; aber Längsschnittsdaten aus Studien mit Messwiederholung sind bekanntlich belastbarer als die Erfahrungen und Beobachtungen einzelner im Querschnitt [38].

Was genau wurde untersucht? - „Unter digitalen Spielen wurden alle Spiele verstanden, die auf digitalen Endgeräten genutzt werden (Smartphone, Tablet, Computer, Spielekonsole). Unter sozialen Medien wurden Messenger-Dienste wie WhatsApp und Threema, Video- und Streaming-Dienste mit Kommentar und/oder Like-Funktion wie YouTube und TikTok, digitale Fotoalben wie In-

1 Wurden vom Unternehmen Netflix selbst zu Anfang des Jahres für das erste Quartal weltweit 7 Millionen Neukunden vorhergesagt, so lag die tatsächliche Zahl - nicht zuletzt zur Überraschung des Unternehmens selbst - im Zusammenhang mit der Corona-Krise bei 15,8 Millionen neuer zahlender Kunden weltweit [24].

2 https://www.tvmovie.de/news/netflix-gratis-diese-filme-und-serienkoennt-ihr-jetzt-kostenlos-sehen-114267; abgerufen am 6.9.2020. stagram und Snapchat, Mikroblogging-Dienste wie Twitter und Facebook sowie Berufsplattformen wie XING und LinkedIn subsummiert" beschreibt Rainer Thomasius, Ärztlicher Leiter des Deutschen Zentrums für Suchtfragen des Kindes- und Jugendalters (DZSKJ) am Universitätsklinikum Hamburg-Eppendorf, im Rahmen der Pressekonferenz am 29. Juli 2020 in Berlin anlässlich der Vorstellung der Ergebnisse der Studie [44]. Nicht berücksichtigt wurden also Netflix und Apple-TV, von dem erst dieses Jahr eingeführten Disney ${ }^{+}$-Kanal gar nicht zu reden.

Was kam heraus? - Zunächst einmal nahm die Häufigkeit der regelmäßigen Nutzung (mindestens einmal pro Woche) von Computerspielen, die sich schon im September 2019 auf sehr hohem Niveau befand, im April 2020 noch etwas zu und stieg von $84 \%$ auf $97 \%$ signifikant. Auch die Häufigkeit der Vielnutzer (tägliche Nutzung) stieg von $40 \%$ auf $54 \%$ signifikant. Die Nutzungszeiten der regelmäßigen Computerspieler betrugen im Herbst 2019 an Schultagen 80 Minuten und an Wochenenden sowie in den Ferien 149 Minuten, was im April 2020 mit 139 Minuten an Schultagen sowie 193 Minuten an Wochenenden und in den Ferien angestiegen war. Als Motiv für das Computerspielen wurde von 9 von 10 Kindern und Jugendlichen Langeweile angegeben. Im Hinblick auf soziale Online-Medien lagen die Daten ähnlich: Die Häufigkeit der regelmäßigen Nutzung nahm von September 2019 (90\%) bis April 2010 (93\%) zu, die der täglichen Nutzung stieg von 66\% auf $75 \%$ signifikant. Die durchschnittlichen Nutzungszeiten der regelmäßigen Nutzer sozialer Online-Medien betrugen im Herbst 2019 an Schultagen 116 Minuten und an Wochenenden sowie in den Ferien 185 Minuten, was im April mit 193 Minuten an Schultagen sowie 241 Minuten an Wochenenden und in den Ferien nochmals signifikant angestiegen war. Auch soziale Online-Medien wurden von der großen Mehrheit der Kinder und Jugendlichen (86\%) zur Bekämpfung von Langeweile genutzt. Für Computerspiele und soziale Online-Medien zusammen ergibt sich damit folgendes Bild ( $\triangleright$ Abb. 1): An Schultagen nahm die mit beidem verbrachte Zeit mit 3 h 15 min im September 2019 auf 5 h 32 min im April 2020 zu, an Wochenenden und in den Ferien von $5 \mathrm{~h} 34 \mathrm{~min}$ im September auf $7 \mathrm{~h}$ und 14 min im April 2020 zu. Wohlgemerkt, Netflix, Apple-TV und Disney ${ }^{+}$sowie das gute alte Fernsehen sind in diesen Zahlen nicht inbegriffen.

Eine Lehrerin an einem Berliner Gymnasium, selbst Mutter zweier kleiner Kinder, schrieb mir nach der Zeit der Schulschließungen (15. Mai 2020) in einer E-Mail das Folgende: „Die Schüler, die ich jetzt für einige wenige Stunden wiedersehen durfte, haben die 7 Wochen fast ausnahmslos spielend vor dem Rechner verbracht. Sie waren im Schnitt 5-10 Kilo schwerer [... und] sie sprechen davon, dass ihnen ihr Sport fehlt, sie aber aus Angst das Haus nicht verlassen haben. Die meisten meiner Schüler sind 17-19 Jahre alt. Ich finde es in diesem Alter besonders bedenklich, die Schüler in digitale Welten abdriften zu lassen. Hier wird auch der Grundstein für Suchtbiografien gelegt, die später schwer zu korrigieren sein werden" [38].

Nach den hier vorgestellten Daten der DAK-Studie dürfte diese Beobachtung kein Einzelfall sein, sondern die Auswirkungen der erfolgten Schulschließungen recht gut beschreiben, denn gut 2 Stunden mehr Inaktivität an Werktagen und knapp 2 Stunden mehr an Wochenenden und in den Ferien sind nach dem vorliegenden Wissen um Risiken und Gefahren für die Gesundheit junger Menschen 


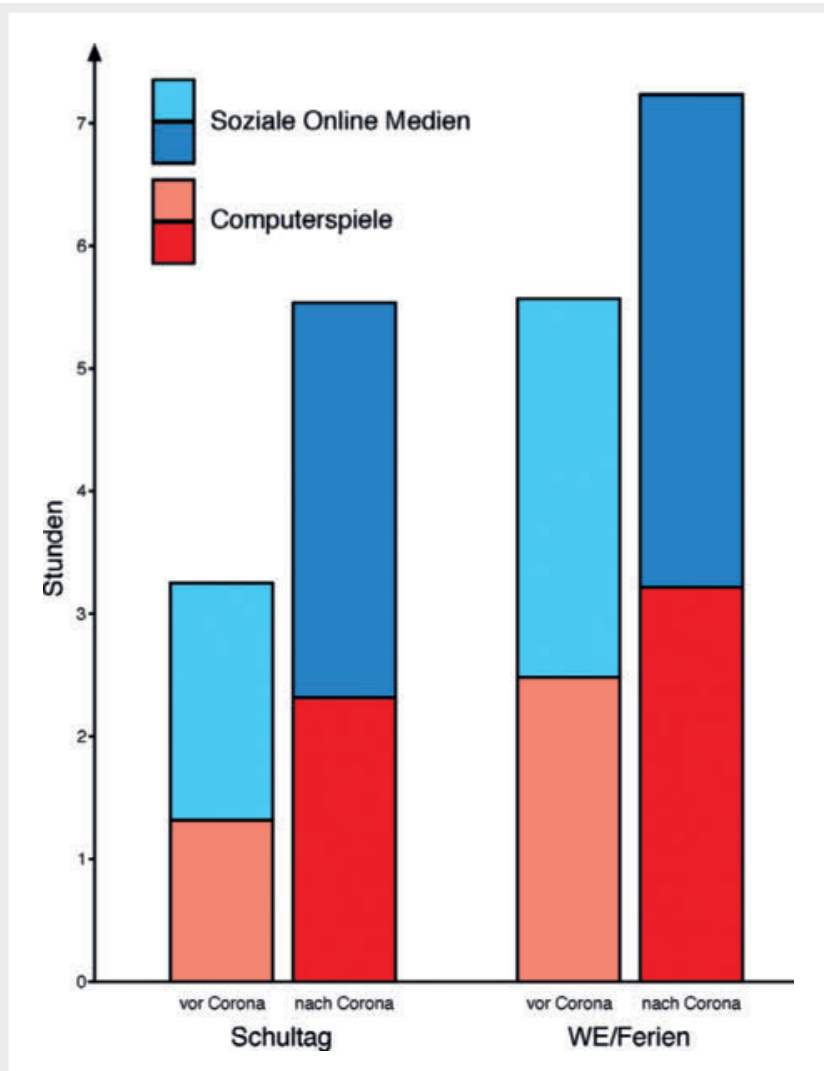

- Abb. 1 Durchschnittliche tägliche Nutzungszeiten (in Stunden) von Computerspielen (rot) und sozialen Online-Medien (blau) durch Kinder und Jugendliche im September 2019 (vor dem Lockdown; heller) und April 2020 (während des Lockdown; dunkler) an Schultagen (links) und Wochenenden bzw. während der Ferien (rechts). Die jeweils korrespondierenden Säulenabschnitte links und rechts unterscheiden sich alle hoch signifikant mit $p<0,001$ (nach Daten aus [44]).

als schwerwiegende Gefährdung einzustufen. Auf diesen Konflikt zwischen Gesundheitsschutz (Infektionsprophylaxe) und Gesundheitsgefährdung (Inaktivität sowie eine Reihe psychischer Probleme) durch den Lockdown weisen mittlerweile auch neueste Publikationen hin [4, 12, 15, 29]. Die gesundheitlichen Auswirkungen von Übergewicht im Kindes- und Jugendalter sind hinlänglich bekannt [27, 33, 34, 43, 46, 47]. Hinzu kommt der leidige Umstand, dass Gewicht viel leichter zu- als abgenommen wird und daher aus übergewichtigen Kindern und Jugendlichen sehr oft übergewichtige Erwachsene werden. Die Folgen - Bluthochdruck und Diabetes (Typ II) - und damit die Langzeitfolgen Schlaganfall, Herzinfarkt und Krebs sind gravierend. So wurden beispielsweise im Jahr 2012 im medizinischen Fachblatt Lancet die weltweiten gesundheitlichen Folgen von körperlicher Inaktivität auf 5 Millionen Todesfälle jährlich beziffert [49]. Darüber hinaus wurde gezeigt, dass eine $10 \%$ ige Reduktion von körperlicher Inaktivität weltweit 533000 Todesfälle pro Jahr abwenden könnte. Anders ausgedrückt: 15-30 Minuten pro Tag zügiges Gehen reduziert das Risiko für Krebs, Herzerkrankungen, Schlaganfälle und Diabetes um 20-30\% und verlängert die Lebenserwartung um 3-5 Jahre. Hinzu kommt, dass sich Übergewicht und mangelnde Bildung gegenseitig bedingen [6].
Auch die Sorgen der Lehrerin im Hinblick auf die Entwicklung einer Sucht sind leider nur zu berechtigt, denn die Corona-Pandemie hat das Problem der Sucht verstärkt $[14,21] .{ }^{3}$ Wurde noch bis vor wenigen Jahren immer wieder angezweifelt, dass es neben den stoffgebundenen Suchterkrankungen auch Verhaltenssüchte gibt, so sind diese (wie schon seit längerer Zeit die Glücksspielsucht) mittlerweile von der WHO als Suchterkrankungen anerkannt und auch ihre Behandlung gefordert. Leider haben Menschen mit Computerspielsucht auch ein höheres Risiko, an stoffgebundenen Süchten zu erkranken, was teilweise darauf zurückzuführen sein dürfte, dass einerseits eine allgemeine, erbliche Neigung zur Sucht beide Suchtformen betrifft und andererseits in Computerspielen nicht selten auch der Substanzgebrauch propagiert wird. Suchtbiografien sind tatsächlich mindestens so schwer zu korrigieren wie „Übergewichtsbiografien“.

Lassen wir nochmals den Autor der aktuellen DAK-Studie zu Wort kommen: „Während des Corona-Lockdowns stiegen die Nutzungsumfänge von digitalen Spielen und sozialen Medien sowohl bei Kindern und Jugendlichen [...] deutlich an. Kinder und Jugendliche mit riskanter bzw. pathologischer Mediennutzung wiesen gegenüber unauffälligen und Nichtnutzern besonders hohe Steigerungsraten bei den Nutzungszeiten auf. Unter Kindern und Jugendlichen, die digitale Spiele und soziale Foren mindestens einmal wöchentlich nutzen, bewegen sich die Zuwächse je nach Gelegenheit und Medium zwischen 29\% und $75 \%$ “ [44].

Nicht nur bei Kindern und Jugendlichen stieg der Medienkonsum während des Lockdowns an, sondern auch bei deren Eltern. Regelmäßiges (mindestens einmal pro Woche) Computerspielen betrieben vor Corona 55,4\% und während Corona 65,8 \% der Eltern, tägliches Computerspielen stieg von 24,3\% auf 33,1 \% hoch signifikant an. Regelmäßig in sozialen Medien waren vor Corona 65,6 \% und während des Lockdown 71,8 \% der Eltern unterwegs. Die Nutzungszeiten von Computerspielen und sozialen Online-Medien zusammen stiegen bei den Eltern werktags von $2 \mathrm{~h} 19$ min vor dem Lockdown auf $3 \mathrm{~h} 35$ min während des Lockdowns und an Wochenenden von $3 \mathrm{~h} 23$ min vorher auf $4 \mathrm{~h} 33$ nachher. Interessanterweise war auch bei den Eltern das Hauptmotiv die Langeweile, bei Computerspielen in $75 \%$ und bei sozialen Online-Medien in $58 \%$ der Fälle. Elterliche Regeln zur Mediennutzung ihrer Kinder gab es in etwa zwei Dritteln der Familien, kontrolliert wurden sie zu etwa $50 \%$ durch die Eltern, unter Corona jedoch etwas weniger.

Es ist schon eigenartig: $90 \%$ der Schüler und $75 \%$ von deren Eltern spielen aus Langeweile ${ }^{4}$ Computerspiele; während des Lock-

3 Nach dem Lockdown häuften sich Berichte aus verschiedenen Ländern über vermehrte Einkäufe von Alkohol (z. B. Rotwein in Frankreich), was sowohl vermehrtes Horten (wie in Deutschland beim Toilettenpapier) als auch auf vermehrten Konsum hinweisen kann. Eine während der Zeit des Lockdowns durchgeführte anonyme Umfrage von Wissenschaftlern des ZI Mannheim an 2102 Personen ergab, dass etwa ein Drittel der Befragten (34,7\%) angaben, seit dem Lockdown mehr oder sogar deutlich mehr Alkohol zu trinken als davor [21]. Auch der illegale Verkauf von Cannabis nahm durch die Corona-Pandemie um 27 \% zu [14].

4 Neben Langeweile werden von Kindern und Jugendlichen auch Realitätsflucht (38\%), Stressabbau (33\%), das Vergessen von Sorgen (30\%) und die Abreaktion von Wut (12\%) als Gründe genannt. Eltern nennen neben Langeweile die Flucht aus der Realität (33\%), den Abbau von Stress (47\%), das Vergessen von Sorgen (29\%) und das Entladen von Wut (6\%). 
down, die Eltern gut eine Stunde und die Kinder gut 2 Stunden täglich mehr als sonst. Computerspiele werden in der Fachwelt - in Anlehnung an die Termini „Hardware“ und „Software“ auch als „Exploitationware“ bezeichnet, also als Instrumente zur Ausbeutung oder wie man heute sagt, zur „Monetarisierung“ von Zeit. Computerspiele sind bekanntermaßen so programmiert, dass sie Sucht erzeugen, sodass bei Langeweile automatisch nach ihnen gegriffen wird. Diese durch Langeweile verursachte Sucht ist letztlich der Grund dafür, dass Langeweile tödlich sein kann. Die uns umbringende Pandemie der Inaktivität [1, 11, 22, 30] ist Teil des Geschäftsmodells der genannten großen Firmen.

Wie anderswo ausgeführt [41], sollte Langeweile jedoch gar nicht bekämpft und zum Gewinn der ohnehin schon reichsten Konzerne der Welt umgemünzt werden, sondern Kreativität in der realen Welt provozieren, um zu einer besseren Entwicklung und schließlich zur Passung der eigenen Neigungen und Fähigkeiten auf die Welt zu gelangen und damit die Frage nach dem Sinn der eigenen Existenz immer wieder neu zu beantworten. In welcher Zeit und in welcher Welt leben wir? - Gehen Sie bitte nochmals die anfangs aufgelisteten Punkte der nachgewiesenen gesellschaftlichen Schäden durch digitale Bildschirmmedien durch. Aufgrund von Corona haben wir von all dem jetzt noch mehr! - Man fühlt sich stark an Aldous Huxley's Utopie „Schöne neue Welt“ erinnert, in der alle süchtig gemacht werden, damit sie nicht merken, in welch miserablem, entmenschlichtem Zustand sie leben. Im Rahmen von Corona wird häufig betont, dass wir nachher nicht so weitermachen wollen und auch nicht sollten wie vorher. Hoffen wir, dass wir das ernst nehmen und uns nachher auch daran erinnern. Nur dann hat „die Geschichte von 2 Pandemien“ [15] einen guten Ausgang.

\section{Literatur}

[1] Andersen LB, Mota J, Di Pietro L. Update on the global pandemic of physical inactivity. Lancet 2016; 388: 1255-1256

[2] Bond RM, Fariss C], Jones J], et al. A 61-million-person experiment in social influence and political mobilization. Nature 2012; 489: 295-298

[3] Briscoe F, Ajunwa I, Gaddis A et al. Evolving public views on the value of one's DNA and expectations for genomic database governance: Results from a national survey. PLoS ONE 2020; 15: e0229044

[4] Brodeur A,Clark AE, Fleche S, et al. Assessing the impact of the coronavirus lockdown on unhappiness, loneliness, and boredom using Google Trends. arXiv: 2004.12129

[5] Brückle M. Netflix und Youtube senken wegen Corona Bildqualität zur Entlastung der Netze https://www.buffed.de/Netflix-Firma-259629/ News/Streaming-Qualitaet-Corona-1345982/; abgerufen am 6.9.2020

[6] Cohen AK, Rai M, Rehkopf DH, et al. Educational attainment and obesity: A systematic review. Obes Rev 2013; 14: 989-1005

[7] DAK. Internetsucht im Kinderzimmer. DAK-Studie: Elternbefragung zur Computernutzung bei 12- bis 17-Jährigen. DAK-Gesundheit. Gesetzliche Krankenversicherung Hamburg 2015

[8] DAK. WhatsApp, Instagram und Co. - so süchtig macht Social Media. DAK-Studie: Befragung von Kindern und Jugendlichen zwischen 12 und 17 Jahren. DAK-Gesundheit. Gesetzliche Krankenversicherung Hamburg 2018

[9] DAK. Geld für Games - wenn Computerspiel zum Glücksspiel wird. Ergebnisse einer repräsentativen Befragung von Kindern und Jugendlichen im Alter von 12 bis 17 Jahren DAK-Gesundheit 2019
[10] DAK. Mediensucht 2020 - Gaming und Social Media in Zeiten von Corona. DAK-Längsschnittstudie: Befragung von Kindern, Jugendlichen (12-17 Jahre) und deren Eltern, https://www.dak.de/dak/meine-familie/mediensucht-bei-kindern-2295590.html\#/) file://Users/ manfredspitzer/Downloads/dak-studie-gaming-social-media-und-corona-2296454\% 20.pdf

[11] Ding D, Lawson KD, Kolbe-Alexander TL, et al. The economic burden of physical inactivity: a global analysis of major non-communicable diseases. Lancet 2016; 388: 1311-1324

[12] Dominski FH, Brandt R. Do the benefits of exercise in indoor and outdoor environments during the COVID-19 pandemic outweigh the risks of infection? Sport Sciences for Health 2020; 16: 583-588

[13] Forsa Politik und Sozialforschung. Game- und Social-Media-Konsum im Kindes- und Jugendalter - Wiederholungsbefragung vor dem Hintergrund der Corona-Krise (Längsschnittuntersuchung). Ergebnisse einer Eltern-Kind-Befragung mit forsa.omninet, 15. Mai 2020. In: DAK (2020) Mediensucht 2020 - Gaming und Social Media in Zeiten von Corona. DAK-Längsschnittstudie: Befragung von Kindern, Jugendlichen (12-17 Jahre) und deren Eltern. DAK Gesundheit 2020

[14] Groshkova T, Stoian T, Cunningham A, et al. Will the Current COVID-19 Pandemic Impact on Long-term Cannabis Buying Practices? J Addict Med 2020; 14. doi:10.1097/ADM.0000000000000698

[15] Hall G, Laddu D, Phillips S, et al. A tale of two pandemics: How will COVID-19 and global trends in physical inactivity and sedentary behavior affect one another? Progress in Cardiovascular Diseases 2020; doi:10.1016/j.pcad.2020.04.005

[16] Holt-Lunstad J, Smith TB, Baker M, et al. Loneliness and social isolation as risk factors for mortality: A meta-analytic review. Perspectives on Psychological Science 2015; 10: 227-237

[17] Holt-Lunstad J, Smith TB, Layton JB. Social relationships and mortality risk: A meta-analytic review. PLoS Medicine 2010; 7(7): e1000316

[18] Huxley A. Schöne Neue Welt. München: Fischer 1932/2014

[19] Johnson NF, Leahy R, Johnson Restrepo N, et al. Hidden resilience and adaptive dynamics of the global online hate ecology. Nature 2019; 573: $261-265$

[20] Kohl HW, Craig CL, Lambert EV, et al. The pandemic of physical inactivity: global action for public health. Lancet 2012; 380: 294-305

[21] Koopmann A, Georgiadou E, Kiefer F, et al. Did the General Population in Germany Drink More Alcohol during the COVID-19 Pandemic Lockdown? Alcohol Alcohol 2020; agaa058, doi:10.1093/alcalc/agaa058

[22] Kramer ADI, Guillory JE, Hancock JT. Experimental evidence of massive-scale emotional contagion through social networks. PNAS 2014; 111: $8788-8790$

[23] Kushlev K, Proulx JDE. The Social Costs of Ubiquitous Information: Consuming Information on Mobile Phones Is Associated with Lower Trust. PLoS ONE 2016; 11: e0162130

[24] Lindner R. Die Welt schaut Netflix. FAZ 21.4.2020 https://www.faz.net/ aktuell/wirtschaft/unternehmen/corona-pandemie-sorgt-fuer-kundenansturm-bei-netflix-16736051.html; abgerufen am 6.9.2020

[25] Matz SC, Kosinski M, Nave G, et al. Psychological targeting as an effective approach to digital mass persuasion. PNAS 2017; 114: 714-12

[26] Müller K, Schwarz C. Fanning the Flames of Hate: Social Media and Hate Crime (November 30, 2018). Social Science Research Network, SSRN. https://ssrn.com/abstract=3082972 oder http://dx.doi. org/10.2139/ssrn.3082972; abgerufen am 9.9.2019

[27] Park M, Falconer C, Viner R, et al. The impact of childhood obesity on morbidity and mortality in adulthood: a systematic review. Obes Rev 2012; 13: 985-1000

[28] Paschke K, Austermann MI, Thomasius R. Assessing ICD-11 Gaming Disorder in Adolescent Gamers: Development and Validation of the Gaming Disorder Scale for Adolescents (GADIS-A). J Clin Med 2020; 9: 993 
[29] Peçanha T, Goessler KF, Roschel H, et al. Social isolation during the COVID-19 pandemic can increase physical inactivity and the global burden of cardiovascular disease. Am J Physiol Heart Circ Physiol 2020; 318: H1441-H1446

[30] Pratt M, Ramirez Varela A, Salvo D, et al. Attacking the pandemic of physical inactivity: what is holding us back? Br J Sports Med 2020; 54: 760-762

[31] Prince J, Wallsten S. How Much is Privacy Worth Around the World and Across Platforms? Technology Policy Institute (TPI), Washington, DC; January 2020//techpolicyinstitute.org/wp-content/uploads/2020/02/ Prince_Wallsten_How-Much-is-Privacy-Worth-Around-the-WorldandAcross-Platforms.pdf; abgerufen am 8.4.2020

[32] Richards R, McGee R, Williams SM, et al. Adolescent screen time and attachment to peers and parents. Archives of Pediatrics \& Adolescent Medicine 2010; 164: 258-262

[33] Roberto CA, Swinburn B, Hawkes C, et al. Patchy progress on obesity prevention: emerging examples, entrenched barriers, and new thinking. Lancet 2015; 385: 2400-2409

[34] Robinson TN, Banda JA, Hale L, et al. Screen media exposure and obesity in children and adolescents. Pediatrics 2017; 140: S97

[35] Romero Starke K, Hegewald J, Schulz A, et al. Cardiovascular health outcomes of mobbing at work: results of the population-based, five-year follow-up of the Gutenberg health study. J Occup Med Toxicol 2020; $15: 15$

[36] Salganik M], Dodds PS, Watts DJ. Experimental study of inequality and unpredictability in an artificial cultural market. Science 2006; 311 : 854-856

[37] Spitzer M. Einsamkeit. München: Droemer 2018

[38] Spitzer M. Pandemie. München: MVG 2020

[39] Spitzer M. Psychologie und Pandemie. Die Auswirkungen des Corona-Virus auf den Einzelnen und auf die Gesellschaft. Nervenheilkunde 2020; 39: 274-283

[40] Spitzer M. Pandemie - historisch, systematisch, kulturell und (sozial-) politisch. Nervenheilkunde 2020; 39: 363-372
[41] Spitzer M. Langeweile ist nicht langweilig. Von einer nebensächlichen Dummheit zur Funktion und Fehlfunktion. Nervenheilkunde 2020; 39: 612-625

[42] Steele NM, Rodgers B, Fogarty G]. The Relationships of Experiencing Workplace Bullying with Mental Health, Affective Commitment, and Job Satisfaction: Application of the Job Demands Control Model. Int J Environ Res Public Healthv 2020; 17: 2151

[43] Streb J, Kammer T, Spitzer M, et al. Extremely reduced motion in front of screens: Investigating real-world physical activity of adolescents by accelerometry and electronic diary. PLoS ONE 2005; 10: e0 126722

[44] Thomasius R. Riskante und pathologische Nutzung von Games und sozialen Medien durch Kinder und Jugendliche nach Kriterien des ICD-11 und Einfluss des COVID-19-Lockdowns auf Medienzeiten in deutschen Familien. In: Mediensucht 2020 - Gaming und Social Media in Zeiten von Corona. DAK-Längsschnittstudie: Befragung von Kindern, Jugendlichen (12-17 Jahre) und deren Eltern. Juli 2020. DAK Gesundheit 2020

[45] Thomasius R. Kernergebnisse der Studie in Grafiken. Gaming und Social-Media - Das Nutzungsverhalten 10- bis 17-Jähriger und ihrer Eltern vor und unter dem Corona-Lockdown. In: Mediensucht 2020 - Gaming und Social Media in Zeiten von Corona. DAK-Längsschnittstudie: Befragung von Kindern, Jugendlichen (12-17 Jahre) und deren Eltern. Juli 2020. DAK Gesundheit 2020

[46] Thomée S, Lissner L, Hagberg M, et al. Leisure time computer use and overweight development in young adults - a prospective study. BMC Public Health 2015; 15: 839

[47] Tremmel M, Gerdtham U-G, Nilsson PM, et al. Economic Burden of Obesity: A Systematic Literature Review. Int J Environ Res Public Health 2017; 14:

[48] Vosoughi S, Roy D, Aral S. The spread of true and false news online. Science 2018; 359; 1146-1151

[49] Wen C P, Wu X. Stressing harms of physical inactivity to promote exercise. Lancet 2012; 380: 192-193

[50] Williams ML, Burnap P, Javed A, et al. Hate in the machine: Anti-Black and anti-muslim social media posts as predictors of off-line racially and religiously aggravated crime. Bristish journal of Criminology 2019; 60: $93-117$

[51] Spitzer M. Der Wert unserer Daten. Nervenheilkunde 2020; 39: 417423 